\title{
Nondiffusive transport regimes for suprathermal ions in turbulent plasmas
}

\author{
A. Bovet, ${ }^{*}$ A. Fasoli, P. Ricci, and I. Furno \\ École Polytechnique Fédérale de Lausanne (EPFL), Centre de Recherches en Physique des Plasmas (CRPP), CH-1015 Lausanne, Switzerland \\ K. Gustafson \\ École Polytechnique Fédérale de Lausanne (EPFL), Laboratory of Computational Systems Biology, \\ Institute of Bioengineering, School of Life Sciences, CH-1015 Lausanne, Switzerland
}

(Received 16 January 2015; published 3 April 2015)

\begin{abstract}
The understanding of the transport of suprathermal ions in the presence of turbulence is important for fusion plasmas in the burning regime that will characterize reactors, and for space plasmas to understand the physics of particle acceleration. Here, three-dimensional measurements of a suprathermal ion beam in the toroidal plasma device TORPEX are presented. These measurements demonstrate, in a turbulent plasma, the existence of subdiffusive and superdiffusive transport of suprathermal ions, depending on their energy. This result stems from the unprecedented combination of uniquely resolved measurements and first-principles numerical simulations that reveal the mechanisms responsible for the nondiffusive transport. The transport regime is determined by the interaction of the suprathermal ion orbits with the turbulent plasma dynamics, and is strongly affected by the ratio of the suprathermal ion energy to the background plasma temperature.
\end{abstract}

DOI: 10.1103/PhysRevE.91.041101

PACS number(s): 52.25.Fi, 05.40.Fb, 52.35.Ra, 52.55.Pi

Diffusion of tracers in a neutral fluid was observed by Brown and explained through collisional theory by Einstein and Smoluchowski [1,2]. Classical diffusive transport, originating from scale-fixed random walks with a typical step size, $\Delta$, and a typical waiting time between steps, $\tau$, leads to a linear scaling of the mean-squared displacement with time and a diffusion coefficient given by $\Delta^{2} / \tau$. In many complex systems such as fusion and space plasmas [3], scale lengths or time scales are not well defined, thus transport cannot be modeled as a classical diffusive process. Generalizations of the classical diffusion model, such as Lévy walks [4-6] or fractional Lévy motion [7], allow introducing power-law distributions of the step sizes or waiting times and long-range temporal correlations, introducing a non-Gaussian and non-Markovian character. These generalizations result in nondiffusive transport characterized by a mean-squared displacement (variance of displacement) of an ensemble of individuals that does not necessarily scale linearly with time: $\left\langle(\boldsymbol{r}(t)-\boldsymbol{r}(0))^{2}\right\rangle \propto t^{\gamma}$, with $\gamma \neq 1$ generally, where $\boldsymbol{r}(t)$ represents the positions of individuals and $\langle\cdot\rangle$ indicates the ensemble average. When $\gamma>1$ or $\gamma<1$, the transport is called superdiffusive or subdiffusive, respectively. For the special case of classical diffusion $\gamma=1$.

Using time-resolved measurements we have recently shown that suprathermal ions are more sensitive to the intermittent turbulent structures in the basic plasma device TORPEX when their energy is smaller [8]. In this Rapid Communication, we present measurements of suprathermal ion transport in TORPEX carried out in three spatial dimensions and with varying input energies. We show that, as the ion energy is increased, the transport varies from subdiffusive to superdiffusive as predicted by numerical simulations.

Although earlier experimental and numerical studies suggest that the transport of suprathermal ions in fusion devices and astrophysical plasmas is generally nondiffusive [9-12],

\footnotetext{
*alexandre.bovet@epfl.ch
}

direct measurements of suprathermal ion transport in these environments are limited by the high temperature of the former and the difficulty to access the latter. In addition, detailed knowledge of the turbulence characteristics and of the background plasma is necessary to realistically model the transport of suprathermal ions. These difficulties are overcome in basic plasma devices thanks to easy diagnostic access and well characterized plasma scenarios. For example, experimental evidence of nondiffusive transport regimes for single energy suprathermal ions in electrostatic waves was measured in a linear basic plasma device [13]. Together with experimentally validated numerical simulations of the turbulent plasma state, the basic toroidal plasma device TORPEX [14] provides an ideal framework to assess fundamental mechanisms of the interaction between suprathermal ions and plasma turbulence leading to nondiffusive transport. The curvature and gradient of the magnetic field, due to the toroidal configuration, generate drifts of the suprathermal ions and a turbulence that is dominated by intermittent field-elongated structures, features that are also observed in tokamak plasmas.

TORPEX is a simple magnetized torus [14] with a major radius $R=1 \mathrm{~m}$ and a minor radius $a=0.2 \mathrm{~m}$ (Fig. 1). Helical magnetic field lines are created by a toroidal magnetic field, $B_{t} \simeq 75 \mathrm{mT}$, combined with a weaker vertical magnetic field $B_{v} \simeq 2 \mathrm{mT}$. A stationary hydrogen plasma is produced and sustained on the inboard (high field side) of the vessel by injecting microwaves in the electron cyclotron range of frequencies. Electron temperature and density are typically $T_{e} \simeq 1-6 \mathrm{eV}, n_{e} \simeq 10^{15}-10^{16} \mathrm{~m}^{-3}$. TORPEX plasmas are characterized by the presence of ideal-interchange modes (with a perpendicular wave number, $k_{\Delta} \simeq 35 \mathrm{rad} \mathrm{m}^{-1}$, and wave number parallel to the magnetic field, $k_{\|} \simeq 0$ ) driven by the magnetic curvature and the pressure gradient [15], generated on the high field side of the vessel. Field-aligned structures termed "blobs" are intermittently generated from the interchange mode and propagate radially outward $[16,17]$. The experimental setup for the production and detection of suprathermal ions, shown in Fig. 1, was specifically designed 


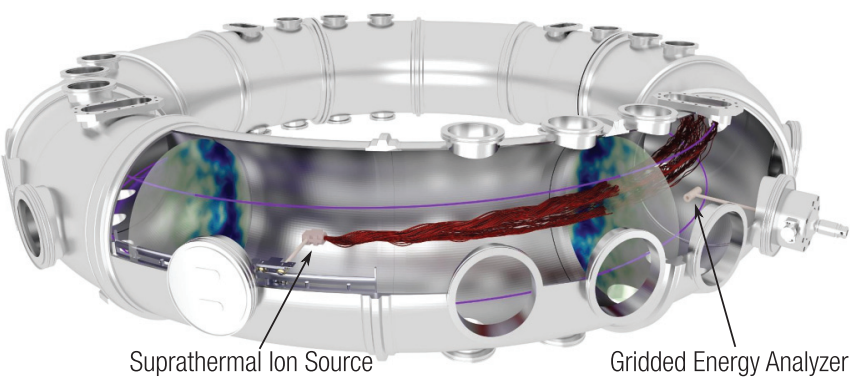

FIG. 1. (Color) View of the TORPEX vessel with magnetic field line and suprathermal ion detection system. TORPEX contains open, helical magnetic field lines (violet) with a radial gradient in field strength that terminate on the vessel. Also shown are the suprathermal ion source on a toroidal sliding track and one detector mounted on a two-dimensional movable system that can also be displaced toroidally. This combination allows measurement of a three-dimensional suprathermal ion current profile. Computed examples of simulated suprathermal ion trajectories with an initial energy of $30 \mathrm{eV}$ are shown in red, emitting from the source. Gyromotion and the irregular spreading of the ion beam due to interaction with the plasma turbulence is apparent. Simulated snapshots of the plasma potential are also displayed at two toroidal positions.

to investigate the suprathermal ion transport properties in a turbulent interchange-mode plasma by measuring the beam spreading. Suprathermal ions are injected in the blob region using a miniaturized lithium $6+$ ion source and detected using gridded energy analyzers $[18,19]$. The source is mounted on a system, enclosed in the vessel, which can move continuously in the toroidal direction. At the injection position, the timeaveraged electron temperature measured by a triple probe is $T_{e} \simeq 1.3 \mathrm{eV}$ [20]. Two detectors capable of moving across almost the entire cross section are installed at different toroidal distances along the suprathermal ion beam. Synchronous detection of the beam is used to improve the signal to noise ratio [19]. Classical collisions with the background plasma are negligible as the slowing-down time of the suprathermal ions due to collisions $\left(\tau_{\mathrm{sd}} \simeq 10 \mathrm{~ms}\right)$ is much larger than their time of flight before detection $\left(\tau_{\mathrm{tf}} \simeq 50 \mu \mathrm{s}\right)$. This setup allows measurements of the time-averaged three-dimensional profile of the suprathermal ion beam as it interacts with the plasma turbulence [19]. In the absence of a plasma, the motion of suprathermal ions in TORPEX is a combination of the gyromotion along the magnetic field lines at the cyclotron frequency, $f_{\mathrm{fi}} \simeq 188 \mathrm{KHz}$, and the upward drift due to the curvature and gradient of the magnetic field. A small spreading in the initial parameters creates a small spreading of the ion Larmor radii and a small initial phase difference in the gyromotion among the ions. This induces an oscillation of the beam width at the cyclotron frequency [18,21]. Figure 2 shows time-averaged suprathermal ion current profiles in the presence of plasma, measured at different toroidal locations for two different energies, $E=30 \mathrm{eV}$ and $E=70 \mathrm{eV}$. On top of the unperturbed motion, broadening due to the interaction with the plasma turbulence is revealed.

The experimental measurements are compared and analyzed with results from numerical simulations with the help of a synthetic diagnostic reproducing the suprathermal ion

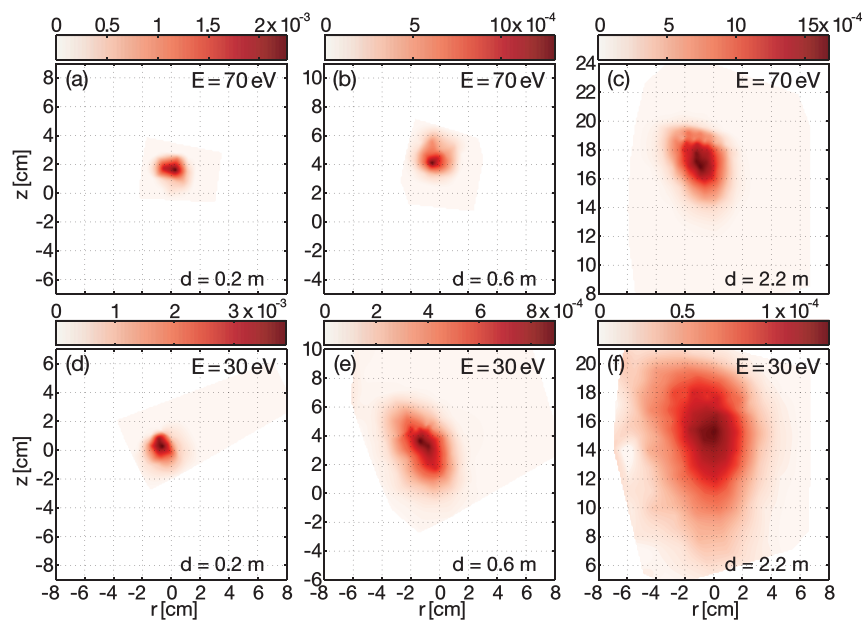

FIG. 2. (Color) Poloidal suprathermal ion current profiles at different toroidal distances [(a), (d) $d=0.2 \mathrm{~m}$; (b), (e) $d=0.6 \mathrm{~m}$; (c), (f) $d=2.2 \mathrm{~m}]$. Top: $E=70 \mathrm{eV}$; bottom: $E=30 \mathrm{eV}$. The vertical drift due to the curvature and gradient of the magnetic field is apparent. The spreading due to the interaction with the turbulent plasmas is revealed and is more important for $30 \mathrm{eV}$ ions. Ion current is stated in $\mathrm{A} / \mathrm{m}^{2}$.

current profile measured with the detectors [19]. In order to identify the transport regime, the radial transport exponent, $\gamma_{R}$, should be computed from the temporal evolution of the variance of the displacement of the suprathermal ions. However, the experimental data is time averaged and the radial spreading of the ions is only accessible as a function of the toroidal distance. Due to the small spreading in the initial injection parameters, the suprathermal ions have slightly different toroidal velocities and there is no unique relation between their time of flight and their toroidal position. For this reason, numerical simulations are necessary to compute the transport exponent. A large number of tracers $\left(1.6 \times 10^{5}\right)$ are injected in first-principles global fluid simulations of TORPEX turbulence [22] that were previously validated against experimental data [23]. Their trajectories are computed using the Newton equation of motion [24].

The comparison between the measured and simulated evolution of the beam width as a function of the toroidal distance is shown in Fig. 3 for the two energies. The radial beam width is computed as the standard deviation of the suprathermal ion current radial profiles. Close to the source, the profiles have a comparable width for the two energies [also shown in Figs. 2(a) and 2(b)]. As the distance from the source is increased, the radial width of the $30 \mathrm{eV}$ ion beam is observed to grow much faster than that of the $70 \mathrm{eV}$ ions. This indicates that the interaction with the plasma turbulence results in a larger spreading for ions with lower energy. A good agreement between the experiments and the simulations is apparent. The toroidal distance between the source and the detector is computed with $1 \mathrm{~cm}$ precision. Statistical error arising from the averaging of the radial standard deviation over the entire profile are also taken into account in the error bars [19].

Figure 4 shows examples of simulated suprathermal ion trajectories on top of a snapshot of the simulated turbulent 


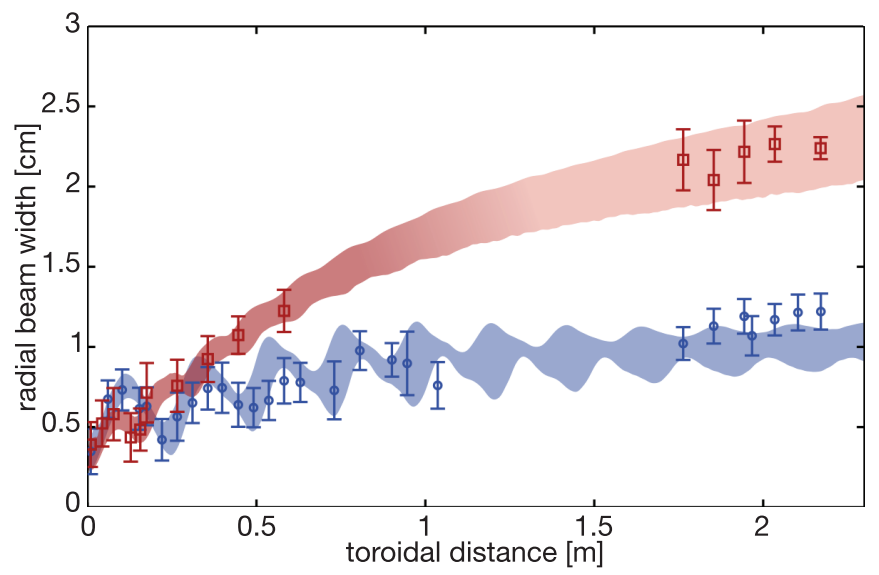

FIG. 3. (Color) Radial width of suprathermal ion current profiles as a function of toroidal distance traveled. Red squares and blue circles represent experimental measurements for ions emitted at 30 and $70 \mathrm{eV}$, respectively. Continuous bands are drawn from a synthetic diagnostic of numerical simulations for $30 \mathrm{eV}$ (red) and $70 \mathrm{eV}$ (blue) ions [19]. The beam width oscillates due to the gyromotion of the ions. A slight discrepancy between measurements and simulations in the phase and amplitude of these oscillations is visible. This comes from the fact that they depend on the injection angle and energy, which can slightly vary from one measurement to the other. The width of the bands is obtained by varying the simulations input parameters within experimental uncertainties. On top of this oscillation, the beam spreads due to the interaction of the ions with the turbulence. The different trends of the spreading indicate different transport regimes. The ballistic phase, lasting approximately one gyroperiod (first three measurements), is in good agreement with the simulations for both energies. Then, the $30 \mathrm{eV}$ ion beam spreads strongly until a toroidal distance of $\simeq 1 \mathrm{~m}$. The accumulated spreading of the $70 \mathrm{eV}$ ion beam is less than the $30 \mathrm{eV}$ beam. Error bars are computed by modeling the measurement with a finite size detector inlet ( $4 \mathrm{~mm}$ radius) and $2 \mathrm{~mm}$ absolute positioning accuracy.

plasma potential, revealing the interplay between the size of the gyro-orbits and that of the turbulent structures. Ions having a smaller initial energy (in red in Fig. 4) have smaller Larmor radii and are more sensitive to turbulent $\mathbf{E} \times \mathbf{B}$ drifts due to the intermittent plasma structures, which can give rise to large radial steps. The numerical simulations show that the step sizes are distributed according to power laws that cannot be associated with a well-defined characteristic length [6,7]. In this case, the transport can be described by a non-Gaussian propagator having heavy tails (a Lévy stable distribution) [7], and is superdiffusive [25,26]. As the energy of the suprathermal ions is increased, their Larmor radii increase (in blue in Fig. 4). When the size of the orbits becomes comparable to the characteristic dimension of the turbulent structures, the suprathermal ions effectively average the turbulent $\mathbf{E} \times \mathbf{B}$ drifts as they perform their gyromotion. Owing to their larger energy, they also experience a faster vertical drift than $30 \mathrm{eV}$ ions, which makes them cross turbulent structures more rapidly in the vertical direction than in the radial direction. Since the turbulence is periodic in the vertical direction, the guiding centers of $70 \mathrm{eV}$ ions often undergo a leftward displacement immediately followed by a rightward displacement, which averages towards zero

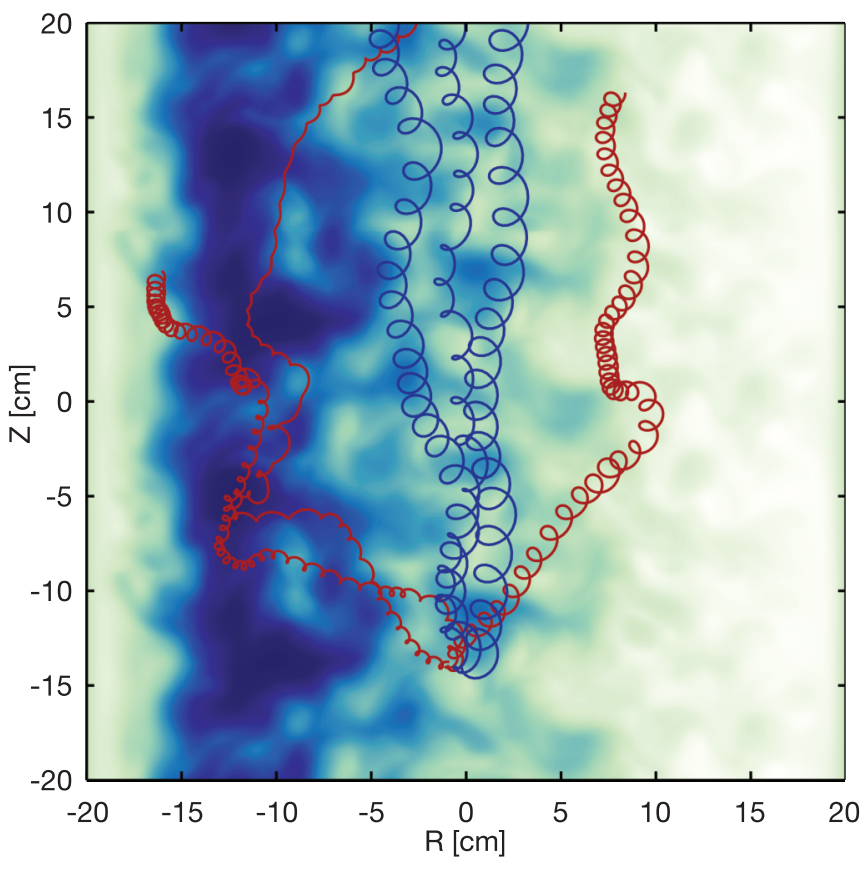

FIG. 4. (Color) Simulated suprathermal ion trajectories projected on the poloidal plane of TORPEX. Ions with an initial energy of $30 \mathrm{eV}$ (in red) have a smaller Larmor radius and are transported by the plasma turbulence. Ions with an initial energy of $70 \mathrm{eV}$ (in blue) have larger Larmor radii and larger vertical drifts. They average the turbulent fluctuations during their gyromotion, which effectively reduces their radial transport. As they drift upward, $70 \mathrm{eV}$ ions perform consecutive steps in opposite radial direction. A snapshot of the simulated plasma potential, displayed in the background, shows the interchange mode positioned approximately at $r=-12 \mathrm{~cm}$ with a vertical wavelength $\lambda \simeq 18 \mathrm{~cm}$ and the turbulent structures propagating toward the low field side.

amplitude during the trajectory. This anticorrelated radial motion is at the origin of subdiffusive transport $[7,26,27]$. Time-resolved measurements in the interaction phase reveal a clear difference between the intermittency level of the suprathermal ion current time traces of the 30 and the $70 \mathrm{eV}$ ions [8]. Superdiffusive ions show a high degree of intermittency, while subdiffusive ones do not, consistently with the picture of a transport governed by heavy-tailed statistics in the former and not in the latter [7]. Conditionally averaged measurements confirm that the intermittency is due to the interaction with the blobs and that the effect of blobs is larger on $30 \mathrm{eV}$ ions than on $70 \mathrm{eV}$ ions [8].

To compute the value of the radial transport exponent, $\gamma_{R}$, the evolution of the variance of the ion radial displacements as a function of time, $\sigma_{R}^{2}(t)$, is computed from the numerical simulations reproducing the experimental conditions (Fig. 5). At first, the transport of the ions is ballistic $\left[\sigma_{R}^{2}(t) \propto t^{2}\right]$, during a phase lasting approximately one gyroperiod. In this short initial phase the ions have not yet interacted with the plasma and are not yet magnetized $[24,28]$. The ions enter a second spreading phase as they start to interact with the plasma turbulence. In this phase, different transport regimes are observed, according to the energy of the ions and the character of the turbulence. A numerical study showed that in 


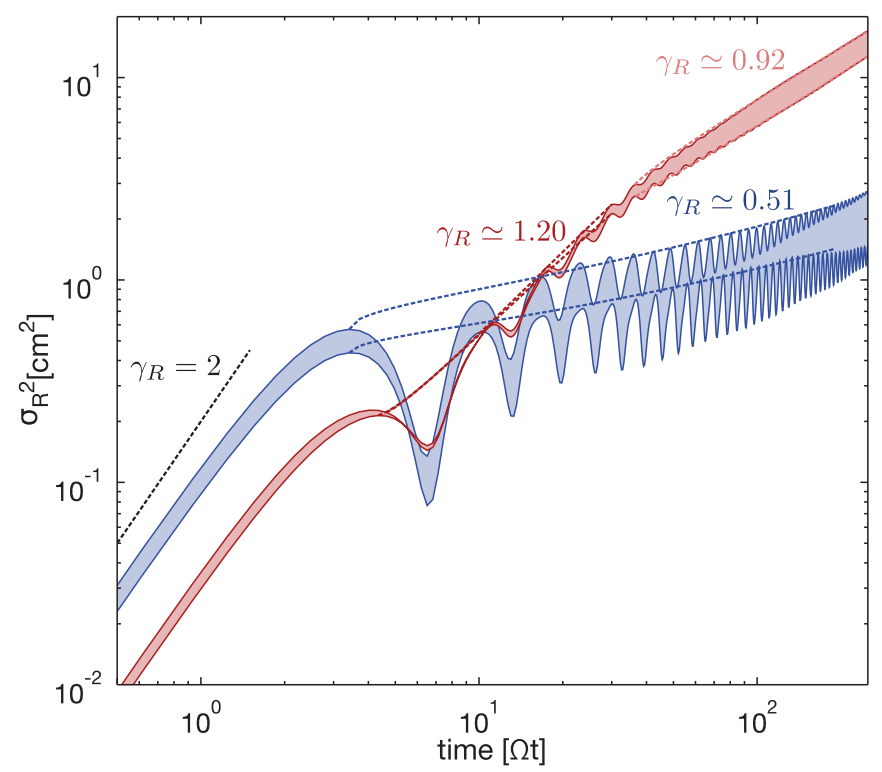

FIG. 5. (Color) Variance of the ion radial positions as a function of time. Results obtained from the numerical simulations reproducing the experimental data in Fig. 3, for ions at $30 \mathrm{eV}$ (red) and $70 \mathrm{eV}$ (blue). Fits of the different phases, shown in dashed lines, provide the values of the transport exponent $\gamma_{R}$. A slope corresponding to $\gamma_{R}=2$ is shown next to the initial ballistic phase. For ions of $30 \mathrm{eV}$, the transport is then superdiffusive with a transport exponent $\gamma_{R} \simeq 1.20$ during approximately four gyromotions and finally close to a diffusive process with $\gamma_{R} \simeq 0.92$. For ions of $70 \mathrm{eV}$, the transport is subdiffusive with $\gamma_{R} \simeq 0.51$. Time is normalized to the ions gyroperiod.

this interaction phase the transport can vary from a subdiffusive to superdiffusive regime depending on two parameters that determine the relative sizes of the ion orbits and the turbulent structures $[6,7,24,27]$ : the injection energy normalized to the electron temperature, $E / T_{e}$, and the normalized fluctuations amplitude, $e \tilde{\phi} / T_{e}$. Fitting the temporal evolution of $\sigma_{R}^{2}$ (Fig. 5) to power laws provides the values of the transport exponents in the different phases. Local maxima of $\sigma_{R}^{2}(t)$ during the gyromotion are fitted with the equation $\sigma_{R}^{2}(t)-\sigma_{R}^{2}\left(t_{0}\right) \propto$ $\left(t-t_{0}\right)^{\gamma_{R}}$, where $t_{0}$ is chosen as the first maximum of each phase. Maxima are chosen since they correspond to the points of the gyromotion that are least affected by orbit effects. This procedure is applied to each simulation used to draw the bands in Fig. 3, and the average is computed for each phase. In the interaction phase, an exponent $\gamma_{R}=0.51 \pm 0.01$ is found for ions of $70 \mathrm{eV}\left(E / T_{e} \simeq 54\right)$ and $\gamma_{R}=1.20 \pm 0.04$ for ions of $30 \mathrm{eV}\left(E / T_{e} \simeq 23\right)$, indicating that the transport varies from subdiffusive to superdiffusive as the energy of the ions is decreased. For ions of $30 \mathrm{eV}$, after the superdiffusive phase, a phase where the transport is close to diffusive $\left(\gamma_{R}=0.92 \pm 0.04\right)$ is visible in Fig. 3 after approximately $1 \mathrm{~m}$ and in Fig. 5 after six gyroperiods. This phase appears when the size of the beam becomes sufficiently large that ions sample regions of the plasma with a different fluctuation amplitude, originating an average transport close to diffusive [27].

We estimate the relative importance of gyroaveraging and drift averaging from the numerical simulations. The conditions identified in the numerical investigations [24,27], for the
TABLE I. Energy $E$, radial transport exponent in the interaction phase $\gamma_{R}$, Larmor radii $\rho_{L}$, ratio of the Larmor orbit diameter to the vertical extend of the blobs, ratio of the vertical to the radial velocity, and ratio of the time required to cross a blob radially to the time required to cross it vertically. All the values are computed from the simulations as the average over all particles and over time. The uncertainties are given by the standard deviation of value distribution for all particles. The Larmor radius is computed from the perpendicular velocity. The vertical velocity is given by the average of the guiding center vertical velocities. The radial velocity is given by the standard deviation of the guiding center radial velocities, since the averaged radial velocity is almost equal to zero.

\begin{tabular}{lccccc}
\hline \hline$E(\mathrm{eV})$ & $\gamma_{R}$ & $\rho_{L}(\mathrm{~cm})$ & $2 \rho_{L} / L_{z}$ & $v_{z} / v_{R}$ & $\tau_{R} / \tau_{Z}$ \\
\hline 70 & $0.51 \pm 0.01$ & $0.8 \pm 0.2$ & 0.6 & $5.5 \pm 0.8$ & 7 \\
30 & $1.20 \pm 0.04$ & $0.5 \pm 0.1$ & 0.3 & $1.5 \pm 0.5$ & 2 \\
\hline \hline
\end{tabular}

gyroaveraging and drift averaging to become effective are

$$
\frac{2 \rho_{L}}{L_{z}}=2 k_{\Delta} \rho_{L}>4
$$

and

$$
\frac{\tau_{R}}{\tau_{Z}}>5,
$$

where, respectively, $\rho_{L}$ is the Larmor radius, $L_{z} \simeq 1 / k_{\Delta} \simeq$ $2.9 \mathrm{~cm}$ is an estimate of the blob vertical extent [27], $L_{R} \simeq \sqrt{L_{p} / k_{\Delta}} \simeq 3.8 \mathrm{~cm}$ is an estimate of the blob radial width [29], and $\tau_{R}$ and $\tau_{Z}$ are the times required for the ions to move radially and vertically across the blobs [27]. Table I shows the Larmor radii, the ratio of the vertical to the radial velocity of the ions, and the parameters indicating the importance of gyroaveraging [Eq. (1)] and drift-averaging [Eq. (2)] for the two injection energies. Both ratios are larger for the $70 \mathrm{eV}$ case than for the $30 \mathrm{eV}$ case indicating the role of these mechanisms in the reduction of the transport for the $70 \mathrm{eV}$ case. The drift-averaging condition of Eq. (2) is satisfied for the $70 \mathrm{eV}$ case revealing the predominant role of the drift-averaging mechanism for $70 \mathrm{eV}$ ions. On the other hand, the gyroaveraging condition [Eq. (1)] is satisfied in neither case. While the estimate of the vertical size of blobs given by $L_{z}$ is in agreement with the value observed in the experiment using conditional sampling [30], it does not take into account the complex structures that constitute a blob. Seeded blob simulations show that, depending on their size, blobs are subject to secondary instabilities [31,32], such as Kelvin-Helmholtz or Rayleigh-Taylor instabilities, that develop in complex shapes with smaller scales than the blob size. These fine structures are not resolved by the conditionally averaged measurements $[8,16,17,30]$. Moreover, different blob sizes are present in TORPEX [16]. The smallest ones are not detected, due to the insufficient resolution of the measurements. The gyroaveraging condition, Eq. (1), is therefore too strict to account for this complexity. As a matter of fact, as the ion Larmor radii increase, the suprathermal ions gradually gyroaverage fine structures and small blobs, resulting in a reduced transport. The transition from the superdiffusive regime to the subdiffusive one is continuous as the energy of the ions, and consequently their Larmor radii, 
increase. Nevertheless, a thorough investigation of the blob size and fine structures in TORPEX is still needed to fully characterize the effect of gyroaveraging.

In summary, we have shown experimental measurements of suprathermal ion transport in a turbulent magnetized plasma in agreement with validated numerical simulations for two different ion energies. The transport of these ions is shown to be consistent with the nondiffusive transport theory that successfully describes the simulations. At first, during approximately one gyroperiod, the transport is ballistic. After this, the spreading of the beam strongly depends on injection energy. Numerical simulations agreeing with these measurements determine the radial transport exponents $\gamma_{R}$ and reveal the basic physical mechanisms leading to super- or subdiffusive behavior. Suprathermal ions with the lower energy follow Lévy-type (non-Gaussian) superdiffusive transport trajectories as they are transported by the turbulent structures. In the same turbulent plasma, suprathermal ions with higher energies average the turbulent electric field more effectively as larger gyroradii and faster vertical drift lead together to anticorrelated, non-Markovian, subdiffusive transport. The time-resolved measurements previously presented in [8] revealed the different effect of blobs on 30 and $70 \mathrm{eV}$ ions, creating a strong difference in the intermittency of the suprathermal ion current. These measurements are in agreement with the different transport regimes discovered here. This work thus demonstrates that the transport of suprathermal ions in turbulent plasmas can exhibit various nondiffusive regimes.

The authors would like to thank F. Avino and J. Loizu for their helpful discussions, the CRPP technical team for their essential support, and P. De Los Rios for his careful reading of the manuscript. Fruitful discussions with members of the team number 297 "Superdiffusive Transport in Space Plasmas and its Influence on Energetic Particle Acceleration and Propagation" at the ISSI are acknowledged. This work was supported in part by the Swiss National Science Foundation.
[1] A. Einstein, Ann. Phys. 17, 16 (1905).

[2] M. von Smoluchowski, Ann. Phys. 326, 756 (1906).

[3] R. O. Dendy, S. C. Chapman, and M. Paczuski, Plasma Phys. Controlled Fusion 49, A95 (2007).

[4] J. Klafter, A. Blumen, and M. F. Shlesinger, Phys. Rev. A 35, 3081 (1987).

[5] R. Metzler and J. Klafter, J. Phys. A: Math. Gen. 37, R161 (2004).

[6] K. Gustafson and P. Ricci, Phys. Plasmas 19, 032304 (2012).

[7] A. Bovet, M. Gamarino, I. Furno, P. Ricci, A. Fasoli, K. Gustafson, D. Newman, and R. Sánchez, Nucl. Fusion 54, 104009 (2014).

[8] A. Bovet, A. Fasoli, and I. Furno, Phys. Rev. Lett. 113, 225001 (2014).

[9] T. Hauff, M. J. Pueschel, T. Dannert, and F. Jenko, Phys. Rev. Lett. 102, 075004 (2009).

[10] W. W. Heidbrink, J. M. Park, M. Murakami, C. C. Petty, C. Holcomb, and M. A. Van Zeeland, Phys. Rev. Lett. 103, 175001 (2009).

[11] S. Perri and G. Zimbardo, Astrophys. J. 750, 87 (2012).

[12] D. Perrone, R. O. Dendy, I. Furno, R. Sanchez, G. Zimbardo, A. Bovet, A. Fasoli, K. Gustafson, S. Perri, P. Ricci, and F. Valentini, Space Sci. Rev. 178, 233 (2013).

[13] S. Zhou, W. W. Heidbrink, H. Boehmer, R. McWilliams, T. A. Carter, S. Vincena, and S. K. P. Tripathi, Phys. Plasmas 18, 082104 (2011).

[14] A. Fasoli, F. Avino, A. Bovet, I. Furno, K. Gustafson, S. Jolliet, J. Loizu, D. Malinverni, P. Ricci, F. Riva, C. Theiler, M. Spolaore, and N. Vianello, Nucl. Fusion 53, 063013 (2013).

[15] P. Ricci and B. N. Rogers, Phys. Rev. Lett. 104, 145001 (2010).

[16] C. Theiler, I. Furno, P. Ricci, A. Fasoli, B. Labit, S. H. Müller, and G. Plyushchev, Phys. Rev. Lett. 103, 065001 (2009).
[17] I. Furno, M. Spolaore, C. Theiler, N. Vianello, R. Cavazzana, and A. Fasoli, Phys. Rev. Lett. 106, 245001 (2011).

[18] A. Bovet, I. Furno, A. Fasoli, K. Gustafson, and P. Ricci, Nucl. Fusion 52, 094017 (2012).

[19] A. Bovet, I. Furno, A. Fasoli, K. Gustafson, and P. Ricci, Plasma Phys. Controlled Fusion 55, 124021 (2013).

[20] C. Theiler, I. Furno, A. Kuenlin, P. Marmillod, and A. Fasoli, Rev. Sci. Instrum. 82, 013504 (2011).

[21] W. W. Heidbrink, H. Boehmer, R. McWilliams, A. Preiwisch, Y. Zhang, L. Zhao, S. Zhou, A. Bovet, A. Fasoli, I. Furno, K. Gustafson, P. Ricci, T. Carter, D. Leneman, S. K. P. Tripathi, and S. Vincena, Plasma Phys. Controlled Fusion 54, 124007 (2012).

[22] P. Ricci and B. N. Rogers, Phys. Plasmas 16, 092307 (2009).

[23] P. Ricci, C. Theiler, A. Fasoli, I. Furno, K. Gustafson, D. Iraji, and J. Loizu, Phys. Plasmas 18, 032109 (2011).

[24] K. Gustafson, P. Ricci, I. Furno, and A. Fasoli, Phys. Rev. Lett. 108, 035006 (2012).

[25] D. del-Castillo-Negrete, B. A. Carreras, and V. E. Lynch, Phys. Rev. Lett. 94, 065003 (2005).

[26] J. A. Mier, R. Sánchez, L. García, B. A. Carreras, and D. E. Newman, Phys. Rev. Lett. 101, 165001 (2008).

[27] K. Gustafson, P. Ricci, A. Bovet, I. Furno, and A. Fasoli, Phys. Plasmas 19, 062306 (2012).

[28] R. Huang, I. Chavez, K. M. Taute, B. Lukić, S. Jeney, M. G. Raizen, and E.-L. Florin, Nat. Phys. 7, 576 (2011).

[29] P. Ricci, B. N. Rogers, and S. Brunner, Phys. Rev. Lett. 100, 225002 (2008).

[30] C. Theiler, I. Furno, A. Fasoli, P. Ricci, B. Labit, and D. Iraji, Phys. Plasmas 18, 55901 (2011).

[31] D. A. DIppolito, J. R. Myra, and S. J. Zweben, Phys. Plasmas 18, 060501 (2011).

[32] F. D. Halpern, A. Cardellini, P. Ricci, S. Jolliet, J. Loizu, and A. Mosetto, Phys. Plasmas 21, 022305 (2014). 